\title{
Modeling Low-coherence Enhanced Backscattering (LEBS) Using Photon Random Walk Model of Light Scattering
}

\author{
Hariharan Subramanian, Prabhakar Pradhan, Young L. Kim, Yang Liu and Vadim Backman \\ Biomedical Engineering Department, Northwestern University, Evanston, IL 60208.
}

\begin{abstract}
Interference effects caused due to the coherent waves traveling in time reversed paths produces an enhanced backscattering (EBS) cone, which is known to be inversely proportional to the transport mean free path length $\left(l_{s}^{*}\right)$ of a scattering media. In biological media, $l_{s}{ }^{*}(0.5-2 \mathrm{~mm}) \gg$ wavelength $\lambda$, results in an extremely small $\left(\sim 0.001^{0}\right)$ angular width of the EBS cone making the experimental observation of such narrow peaks to be difficult. Hence, we developed a low coherence enhanced backscattering (LEBS) technique by combining the EBS measurements with low spatial coherence illumination and low temporal coherence detection. Low spatial coherence behaves as a spatial filter preventing longer path lengths and collects photons undergoing low orders of scattering. The experimental angular width of these LEBS peaks $\left(\sim 0.3^{0}\right)$ are more than 100 times the width of the peak predicted by conventional diffusion theory. Here we present a photon random walk model of LEBS cones obtained using Monte Carlo simulation to further our understanding on the unprecedented broadening of the LEBS peaks. In general, the exit angles of the scattered photons are not considered while modeling the time reversed interference phenomenon in diffusion regime. We show that these photon exit angles are sensitive to the low orders of scattering, which plays a significant role in modeling LEBS peaks when the spatial coherence length of the light source is much smaller than $l_{s}{ }^{*}$. Our results show that the model is in good agreement with experimental data obtained at different low spatial coherence illumination.
\end{abstract}

Key words: Monte Carlo simulation, Low coherence enhanced backscattering, exit angles, spatial coherence, transport mean free path.

\section{INTRODUCTION}

The constructive self-interference effect due to the coherent waves traveling time-reversed paths in a disordered medium produces an enhanced intensity cone in directions close to the backscattering. In case of complete diffusion of light, the amplitude of this intensity profile as a function of the backscattering angle can be as high as twice that of the incoherent background. ${ }^{1}$ This increase in reflectivity in the backward direction leads to a reduction in the amount of light transported in the forward direction thereby resulting in the weak localization of photon. This phenomenon of enhanced backscattering (EBS, also known as coherent backscattering) was first experimentally observed in aqueous suspensions of polystyrene micrsospheres..$^{1-3}$ In order to explain the enhanced backscattering, typically the light is assumed to be completely spatially coherent. ${ }^{4,5}$ In a homogeneous semi-infinite disordered medium, the full angular width at halfmaximum (FWHM), $\omega_{h m}$, of the EBS cone was shown to be inversely proportional to the ratio of the wavelength of light $\lambda$ to the transport mean free path length $l_{s}{ }^{*}$ of light in the medium: ${ }^{2,3}$

$$
\omega_{h m}=\lambda /\left(3 \pi l_{s}^{*}\right) \text {. }
$$

Although EBS enhancement has been widely studied in a variety of disordered media with relatively short $l_{s}^{* 6,7}$ investigation of EBS in weakly scattering media with $l_{s}^{*}>>\lambda$ has been exceedingly difficult, in part due to very small widths of EBS peaks predicted in such media (e.g., $\omega_{h m} \sim 0.001^{\circ}$ for $l_{s}{ }^{*} \sim 1 \mathrm{~mm}$ ) and excessive speckle. In particular, a biological tissue is one important example of a weakly scattering medium with long $l_{s}{ }^{*}$. Measurement of light scattering and absorption properties of tissue is crucial to exploit the use of light for both diagnostic and therapeutic purposes. ${ }^{8-11}$ Accordingly, EBS may be used as one of the potential tools for noninvasive optical characterization of tissue. However, only very few studies ${ }^{9-11}$ have actually attempted EBS measurements in tissue. In particular, Alfano et al. ${ }^{10,11}$ first reported EBS in biological tissue using femtosecond-time-resolved measurements.

Complex Dynamics and Fluctuations in Biomedical Photonics III, edited by Valery V. Tuchin Proc. of SPIE Vol. 6085, 608503, (2006) · 1605-7422/06/\$15 · doi: 10.1117/12.646868 
Recently, we demonstrated the feasibility of observing EBS under low spatial coherence illumination (spatial coherence length $L_{s c}<<l_{s}{ }^{*}$ ). Low spatial coherence behaves as a spatial filter that rejects longer path-lengths with exponentially low probability, thus, resulting in more than 100 times increase in the angular width of low coherence EBS (LEBS) cones. ${ }^{12,13}$ Furthermore, we showed that not only does LEBS represent a novel enhanced backscattering phenomenon, but it also opens up the feasibility of studying enhanced backscattering in biological tissue and other media with long $l_{s}^{*}$ and enables depth-selective spectroscopic tissue characterization. ${ }^{14}$ For example, we demonstrated that LEBS can be used to diagnose the earliest, previously undetectable stage of colon carcinogenesis that precedes currently histologically detectable lesions. ${ }^{12,13}$ These results underline the need for thorough understanding of this new effect.

Previously, LEBS was observed by combining EBS measurements with low spatial coherence (LSC) illumination and low temporal coherence detection. This technique uses a broadband continuous wave xenon lamp to achieve low spatial coherence illumination. We also demonstrated that the angular width of an EBS peak observed under LSC illumination $\left(\sim 0.3^{\circ}\right)$ is more than 100 times broader than that of conventional EBS. ${ }^{12}$ We note that one of the most intriguing properties of LEBS is the dramatically increased angular width of the LEBS peaks, which cannot be explained on the basis of conventional diffusion approximation based model of EBS alone. In order to further our understanding of this unprecedented broadening of LEBS peaks and identify the origin of LEBS, it is necessary to develop a rigorous model of LEBS. In this manuscript, we model for the first time LEBS using Monte Carlo simulations, show that the model is in excellent agreement with experimental data, and explain the origin of LEBS broadening. We demonstrate that the exit angle of the scattered photons, typically neglected in modeling conventional EBS peaks, is of critical importance when the spatial coherence length of the light source is much smaller than $l_{s}^{*}$ (i.e., $L_{s c}<<l_{s}^{*}$ ). On the other hand, we show that the exit angle of photons plays only a minimal role in the diffusion regime when the spatial coherence length is greater than $l_{s}^{*}$ of the medium (i.e., $L_{s c}>>l_{s}^{*}$ ).

\section{MONTE CARLO MODEL OF LEBS}

In order to model low-coherence enhanced backscattering, we developed a photon random walk model using Monte Carlo (MC) simulation. EBS originates from constructive interference between any given light path and its time reversed counterpart related by the reciprocity theorem. MC simulation provides a distribution of the photon backscattering intensity as a function of radial distance and exit angle for relevant optical parameters. The shape of the EBS cone versus the scattering angle is calculated from the Fourier transform of the radial intensity distribution on the surface of the sample illuminated by a point source simulated by the Monte Carlo method. This technique has been successfully used in modeling EBS in non-biological and biological samples. ${ }^{15-17}$ The intensity of the EBS cone thus obtained in the backward direction, $I_{E B S}$ can be written as,

$$
I_{E B S}\left(\vec{q}_{\perp}\right)=\iint P(r) \exp \left(i q_{\perp} . r\right) d^{2} r
$$

where $P(r)$ is the probability of radial intensity distribution of EBS photons with the radial vector $r$ perpendicular to the incident light and $q_{\perp}$ is the projection of wave vector onto the orthogonal plane in the backward direction. In an isotropic disordered medium, the two dimensional Fourier integral can be further simplified to, ${ }^{13}$

$$
I_{E B S}\left(\vec{q}_{\perp}\right) \propto \int r P(r) \exp \left(i q_{\perp} . r\right) d r
$$

where $r P(r)$ is the radial intensity distribution of the conjugated time-reversed paths around a point-like light source illuminating a sample and the projection of wave vector, $q_{\perp}=2 \pi \sin \theta / \lambda$.

In contrast to conventional EBS, low coherence enhanced backscattering is observed using a broadband light source with low spatial coherence. ${ }^{18}$ Therefore, in order to model the effect of low spatial coherence length of illumination on EBS, we incorporated additional coherence length dependent weighting factor to the numerical simulation. We used the readily derived form of the degree of spatial coherence $C_{L_{\mathrm{cc}}}(r)$ as follows: ${ }^{18}$

$$
C_{L_{s c}}(r)=2 J_{1}\left(r / L_{s c}\right) /\left(r / L_{s c}\right) \text {, }
$$

where $J_{1}$ is the first order Bessel function, $L_{s c}$ is the spatial coherence length corresponding to the $88^{\text {th }}$ percentile of the ideal value of unity, and $r$ is the radial vector perpendicular to the incident light. Thus, the modified LEBS intensity in the presence of a low coherence source can be written as

$$
I_{L E B S}(\theta) \propto \int r P(r) \cdot C_{L_{s c}}(r) \exp \left(i \frac{2 \pi \sin \theta}{\lambda} r\right) d r .
$$


The first term in the above equation, $r P(r)(\equiv I(r))$, is the radial intensity distribution, of the point like source illuminating the sample, which can be obtained using the Monte Carlo simulation.

In our Monte Carlo simulation, approximately $10^{10}$ photon packets are launched into the sample. The optical parameters of the sample are assigned based on the sample size used in the LEBS experiment. ${ }^{12}$ The samples are slabs with infinite lateral extents and having optical parameters of biological relevance $\left(l_{s}^{*} 500 \mu \mathrm{m}--2000 \mu \mathrm{m}, \mathrm{g} \sim 0.6-0.9\right.$, $\lambda=520 \mathrm{~nm}$ ). The radial and angular resolution of the grid system used in the Monte Carlo simulation to collect the low order scattering photons is specified as $1 \mu \mathrm{m}$ and $0.3^{\circ}$ respectively. Once the reflectance probability $P(r)$ is calculated from the Monte Carlo simulation, Eq. (5) is then used to obtain the LEBS peak using the Fourier transform of the radial intensity distribution. The LEBS peak obtained is then convoluted with the angular response of the instrument $\left(\sim 0.04^{\circ}-\right.$ $0.3^{\circ}$ ) to compensate for the finite point-spread function of the detection system and the slight divergence of the incident beam. The width of the convoluted LEBS peak is then compared with those obtained from the LEBS experiment.

\section{RESULTS}

We first obtain the radial intensity distributed on the surface of the sample with $l_{s}{ }^{*}=2 \mathrm{~mm}$. The absorption coefficient $\mu_{a}$ and scattering coefficient $\mu_{s}$ are fixed at $0.0001 \mathrm{~mm}^{-1}$ and $5 \mathrm{~mm}^{-1}$ (at $\lambda=520 \mathrm{~nm}$ ) respectively. The radial intensity distribution is calculated from the Monte Carlo simulation at different exit angles between $0.3^{\circ}$ and $90^{\circ}$. The intensity profile at 3 different exit angles $\left(1.5^{\circ}, 20^{\circ}\right.$ and $\left.80^{\circ}\right)$ is shown in Fig. 1. The radial and angular resolution of the grid system used in the Monte Carlo simulation to collect the diffusive multiple scattering photons is specified as $10 \mu \mathrm{m}$ and $0.3^{\circ}$ respectively. Typically, in diffusion regime, the EBS peak is obtained directly from the Fourier transform of the radial intensity without taking into account the exit angle of the photons.

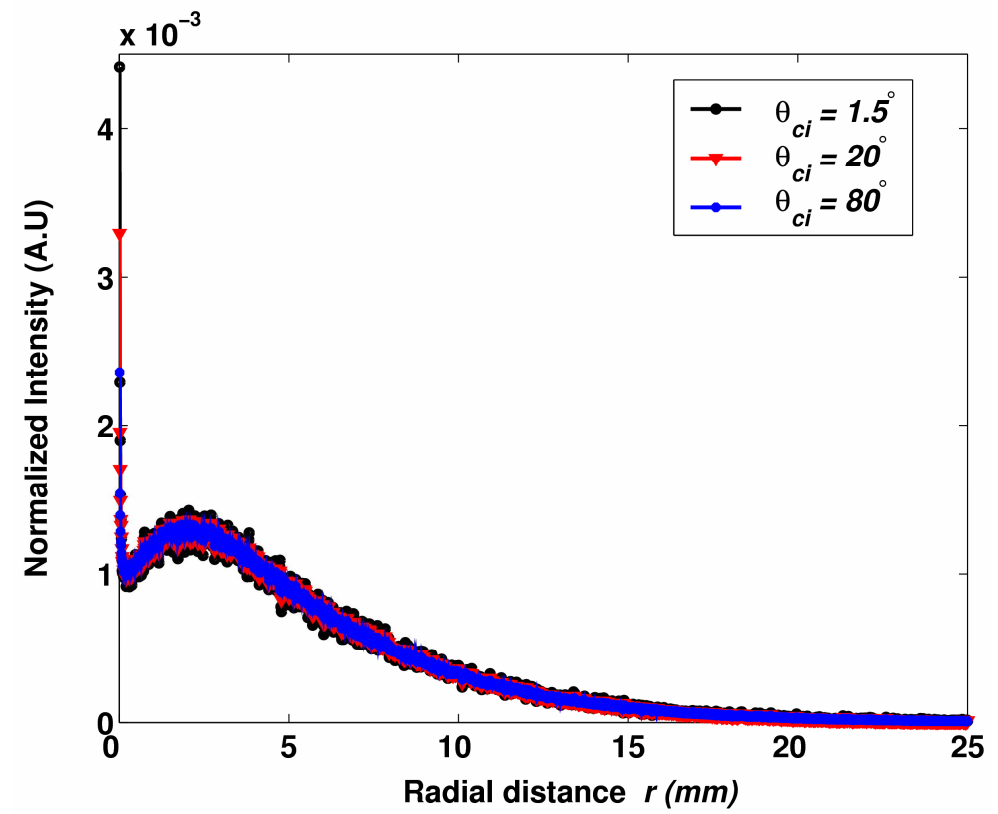

Figure 1: Normalized intensity at different exit angles, $\theta_{c i}$ as a function of radial distance $r$ calculated using Monte Carlo simulation in the diffusive multiple scattering regime $\left(L_{s c}>>l_{s}^{*}\right)$

From Fig. 1, it can be seen that the intensity profile for diffusive multiple scattering remains constant for different exit angles. This intensity profile obtained above can be written as,

$$
\int_{r=0}^{\infty} \int_{\theta=0}^{\pi / 2} p(r, \theta) d r d \theta=N
$$

(for $\mu_{a}=0$ and transmission $=0$ ), where $N$ is the total number of photons injected. Let us now redefine the above probability integral for any finite angle as follows: 


$$
\int_{r=0}^{\infty} \int_{\theta=0}^{\theta c i} p(r, \theta) d r d \theta=\int_{r=0}^{\infty} \bar{p}_{\theta_{c i}}(r) d r=N_{\infty, c i} .
$$

Now we normalize the above probability along the radial direction keeping the maximum angles $\theta_{c i}(\mathrm{i}=1,2,3, .$.

fixed.

Let, $\bar{p}_{\theta_{c i}}(r) / N_{\infty, c i}=P_{\theta_{c i}}(r)$, thus,

$$
\int_{0}^{\infty} P_{\theta_{c i}}(r) d r=1 .
$$

We now consider three different angles, $\theta_{c 1}=1.5^{\circ}, \theta_{c 2}=20^{\circ}$, and $\theta_{c 3}=80^{\circ}$. The shape of the $P_{\theta_{c i}}(r),(\mathrm{i}=1,2$, and 3) curves remains constant, which is shown in Fig. 1. However, a different picture emerges in the case of low spatial coherence illumination when the number of scattering events is restricted using low spatial coherence light source. $P_{\theta_{c i}}(r)$ becomes broader as $\theta_{c i}$ increases from $1.5^{\circ}$ to $80^{\circ}$, as shown in Fig. 2 . This result indicates the importance of considering the exit angles of photons particularly for modeling low coherence enhanced backscattering peaks.

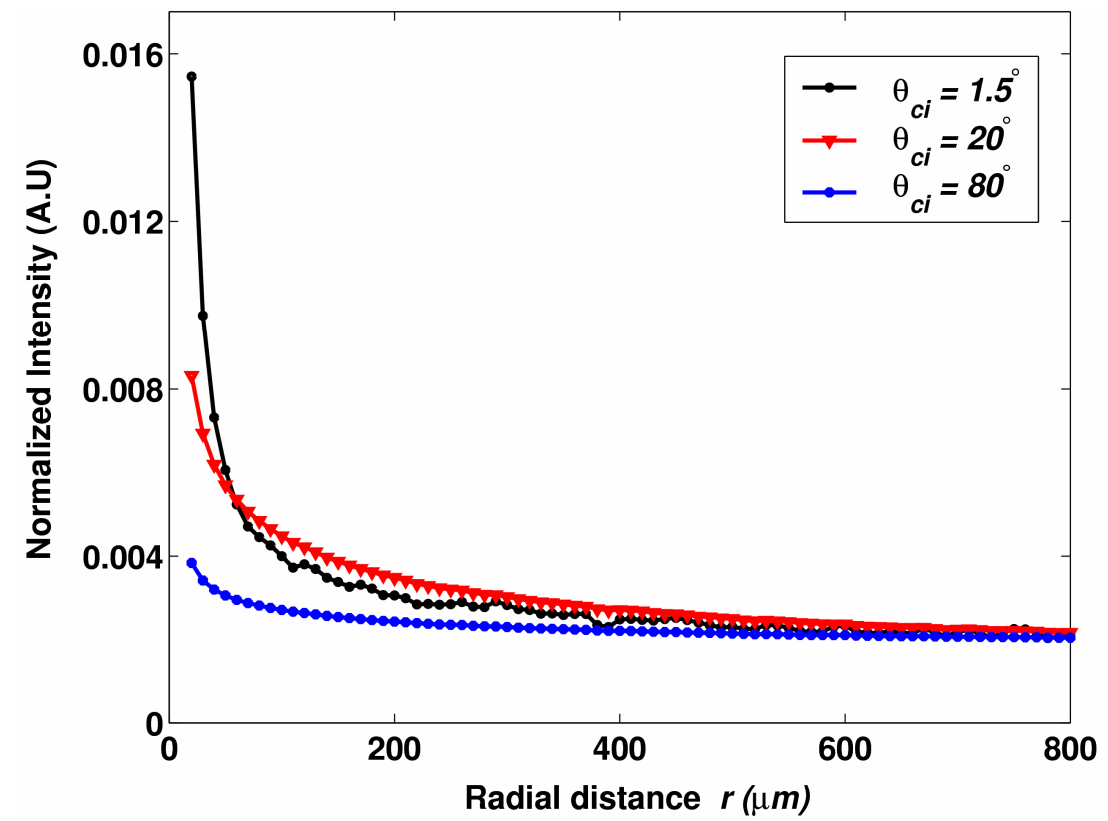

Figure 2: Normalized intensity at different exit angles, $\theta_{c i}$ as a function of radial distance $r$ calculated using Monte Carlo simulation in the low order scattering regime $\left(L_{s c}<<l_{s}{ }^{*}\right)$ when the number of scattering events is restricted due to the finite spatial coherence area.

The exit angle of photons in the image plane is of critical importance for low coherence enhanced backscattering as LEBS probes low orders of scattering in a diffusive multiple scattering medium. This is due to the fact that the trajectories of the photons are clustered into a compact locus to exit at small angles for low orders of scattering, while the trajectories of the photons are less compact for higher orders of scattering. As can be seen from our simulations, the exit angle of the photons is also sensitive to the depth from which the LEBS measurements are obtained (result not shown). As discussed above, the exit angles are less sensitive in the multiple scattering regime providing similar EBS peaks for different exit angles. In diffusion regime, the slight changes in reflectance probability $P(r)$ obtained from Monte Carlo simulation at different exit angles are translated over a radial distance of around $50 \mathrm{~mm}$. Hence these small changes do not translate into narrow/broad peak when the Fourier transform of $P(r)$ is performed.

In order to model LEBS, it is necessary to accurately determine the angle at which the photons are collected. The LEBS signals obtained from simulation are collected at $\theta_{c i} \sim 1.5^{\circ}$ which is close to the width of the experimentally recorded LEBS peaks. This is due to the fact that the angular range of the photons traveling in the time reversed direction 
restricts the exit angle of the photons in the LEBS experiments. It can be seen from Fig. 3 that the probability of exit angle, $P_{e x}\left(\theta_{c i}\right)$ remains almost identical at small angles around $0.3^{\circ}-3^{\circ}$ when spatial coherence light source is used. Therefore, we take the mid-point of this distribution $\left(\sim 1.5^{\circ}\right)$ at small angles for better averaging of $P(r)$. The LEBS peak width obtained from exit angles, $\theta_{c i} \sim 1.5$ degrees deviates less than $5 \%$ when compared to the photons collected at low exit angles $\left(0.3^{\circ}\right.$ to $\left.1^{\circ}\right)$. However, any change in the exit angle of the photons beyond this would significantly change the depth from which the time reversed photons are obtained leading to erroneous modeling of LEBS. We obtain $P(r)$ at $1.5^{\circ}$ using Monte Carlo simulation and then multiply it by the spatial coherence function (Eq. 4) corresponding to the coherence length used in the experiment. The Fourier transform of this multiplied product is then taken to obtain LEBS peak. In subsequent sections, we present detailed validation of the LEBS peak obtained using Monte Carlo simulation by comparing with analytical model and LEBS experiments.

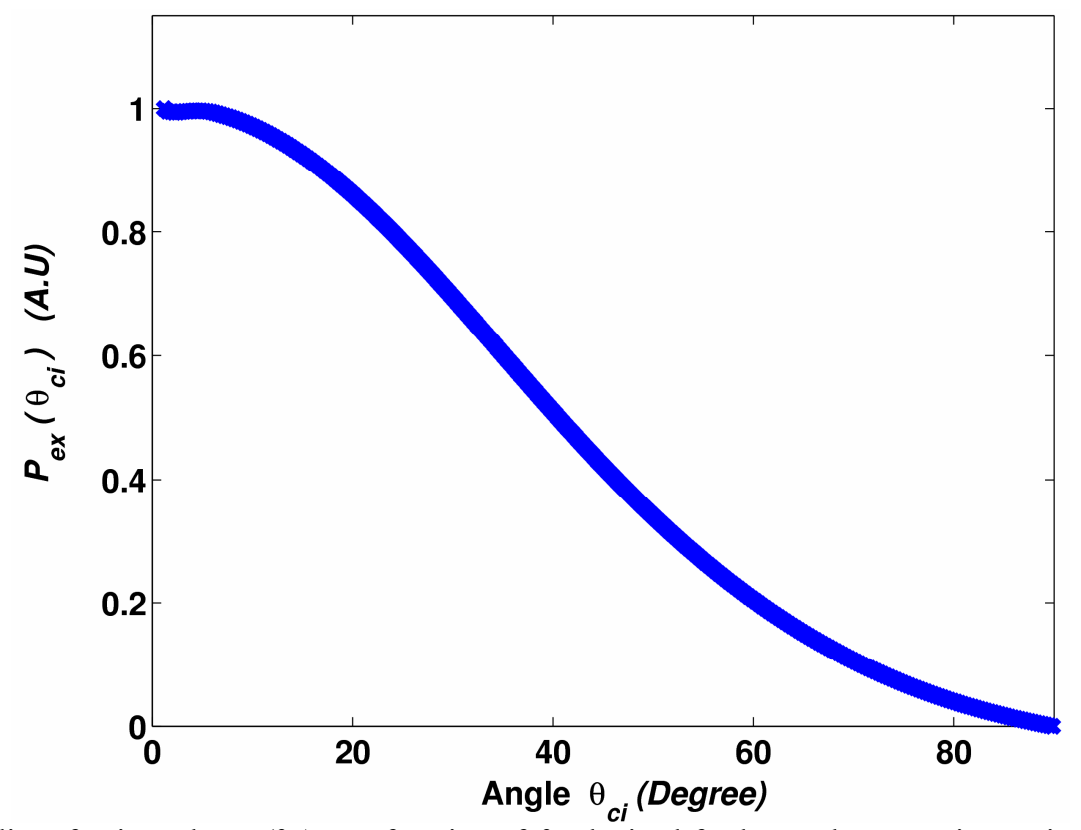

Figure 3: Probability of exit angle $P_{e x}\left(\theta_{c i}\right)$ as a function of $\theta_{c i}$ obtained for low order scattering regime. $P_{e x}\left(\theta_{c i}\right)$ converges at small angles of around $1^{\circ}-3^{\circ}$ when $L_{s c}<<l_{s}^{*}$.

In order to verify the accuracy of the Monte Carlo simulation, we first compare the profile of the EBS peak obtained from numerical simulation with the profile of the EBS peak calculated by Akkermans et al ${ }^{19}$ for an isotropic scattering medium. The simulation is performed with $l_{s}^{*}=2 \mathrm{~mm}$ and infinite spatial coherence $\left(L_{s c}=50 \mathrm{~mm}\right)$, with the exit angle, $\theta_{c i} \approx 1.5^{\circ}$. Figure 4 shows that the simulation results match well with the analytical results. In order to verify the effect of exit angles in EBS measurements, the simulations from small exit angles, $\theta_{c i} \approx 1.5^{\circ}$ are compared to those obtained from higher exit angles (e.g. $\theta_{c i}=80^{\circ}$ ). As shown in Fig. 4, the Monte Carlo simulations for smaller exit angle (circles) agrees with the results obtained from large exit angles (asterisks).

We next validate the spatial coherence function (Eq. 4) used in simulating the LEBS peak by comparing the profiles obtained from the simulation with those of the LEBS experiments (discussed later) for a sample with $l_{s}{ }^{*}$ smaller than $L_{s c}$. In this regime $\left(l_{s}{ }^{*}<<L_{s c}\right)$, though $L_{s c}$ is small, LEBS profiles are primarily determined by $l_{s}{ }^{*}$ as the light scattering paths are not affected by the $L_{s c}$ and hence the role of the finite $L_{s c}$ is virtually insignificant. We simulate the LEBS peak with $L_{s c}=160 \mu \mathrm{m}$ from a sample of $l_{s}^{*}=4 \mu \mathrm{m}$. The LEBS peak in the experiment is collected from white paint (Benjamin Moore) with $l_{s}{ }^{*}=4 \mu \mathrm{m}$ under low spatial coherence illumination (Xenon lamp). As shown in Fig. 5 the LEBS peak obtained from Monte Carlo simulation matches well with the experimentally observed LEBS peak for $l_{s}{ }^{*}<<$ $L_{s c}$. This validates the use of spatial coherence function in the modeling of LEBS peak for low spatial coherence illumination. We further compare the LEBS profile with the EBS peak obtained from infinite spatial coherence length $\left(L_{s c}=50 \mathrm{~mm}\right)$. As expected, the EBS and LEBS peaks are completely indistinguishable as the peak width is completely determined by the small $l_{s}{ }^{*}$ of the medium (Fig. 5). In the next section, we present the experimental verification of the LEBS simulation in the low order scattering regime $\left(L_{s c}<<l_{s}^{*}\right)$. 


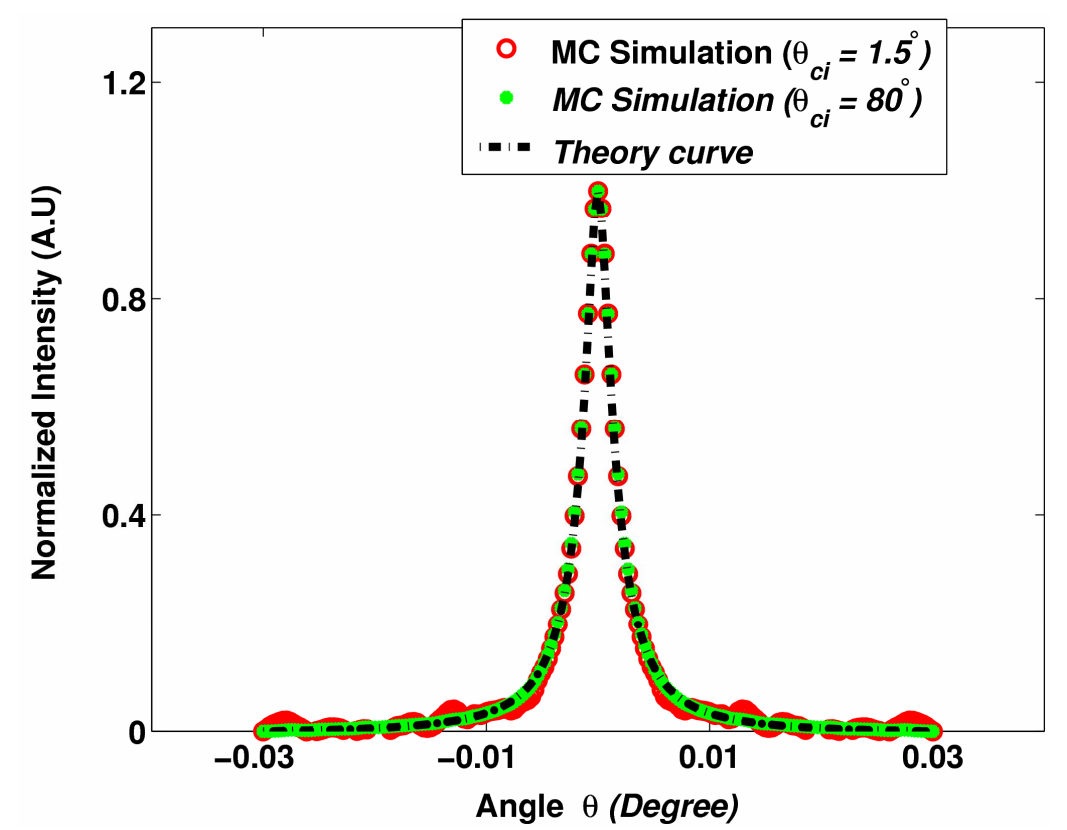

Figure 4: Comparison of the EBS profile from Monte Carlo simulation with that from analytical formulation. ${ }^{19}$ The results from simulation are in excellent agreement with the analytical results. Also, the LEBS simulation for $\theta_{c i}=1.5^{\circ}$ agrees well with the results obtained from $\theta_{c i}=80^{\circ}$ as the width of the EBS peak is insensitive to the $\theta_{c i}$ in the diffusive multiple scattering regime $\left(L_{s c}>>l_{s}{ }^{*}\right)$.

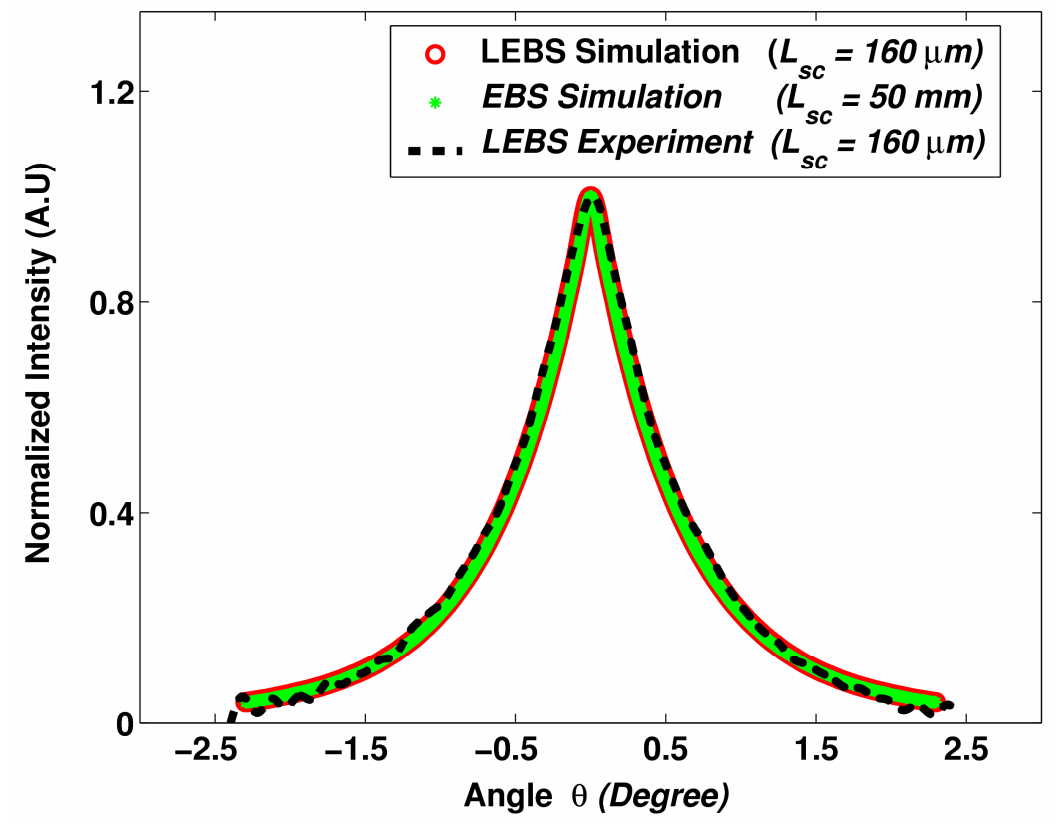

Figure 5: Normalized intensity profile of the LEBS peak as a function of $\theta$ from Monte Carlo simulation is compared with that of the experimental result from white paint under low spatial coherence illumination (Xenon lamp, $\lambda=520 \mathrm{~nm}, L_{s c}=160 \mu \mathrm{m}$ ). The simulation results agrees well with experimentally observed LEBS peak when $L_{s c}=160 \mu \mathrm{m}$. Also, the EBS peak from $L_{s c}=50 \mathrm{~mm}$ and LEBS peak from $L_{s c}=160 \mu \mathrm{m}$ are completely indistinguishable as the peak width is completely determined by the small $l_{s}{ }^{*}$ of the medium and the spatial coherence length plays an insignificant role in this regime.

The LEBS peak obtained using Monte Carlo simulations are experimentally verified. The detailed description of our experimental setup is given elsewhere. ${ }^{12}$ We record LEBS from aqueous suspensions of polystyrene microspheres 
(Duke Scientific, Palo Alto, CA) of various diameters from $200 \mathrm{~nm}$ to $890 \mathrm{~nm}$. The dimension of the samples is $\pi \times 50^{2}$ $\mathrm{mm}^{2} \times 100 \mathrm{~mm}$.

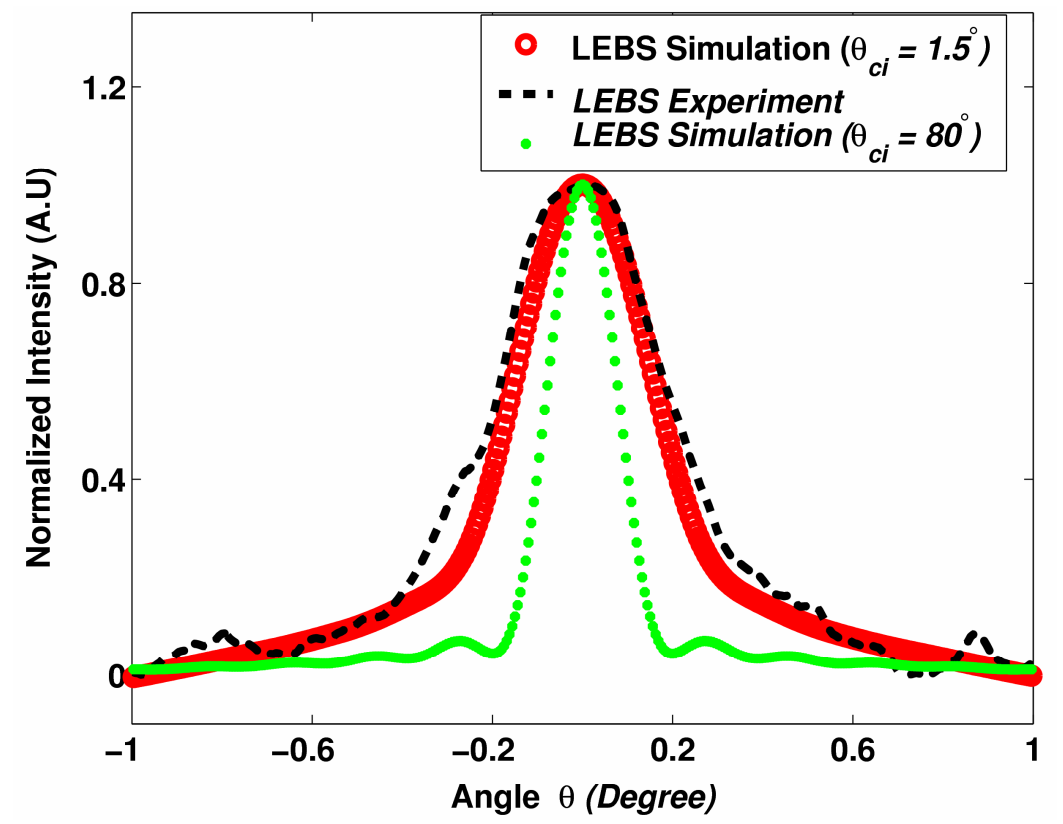

Figure 6: Normalized intensity profile of the LEBS peak as a function of angle $\theta$ from Monte Carlo simulation is compared with that of the aqueous suspensions of polystyrene microspheres (diameter $=0.89 \mu \mathrm{m})$ under low spatial coherence illumination.

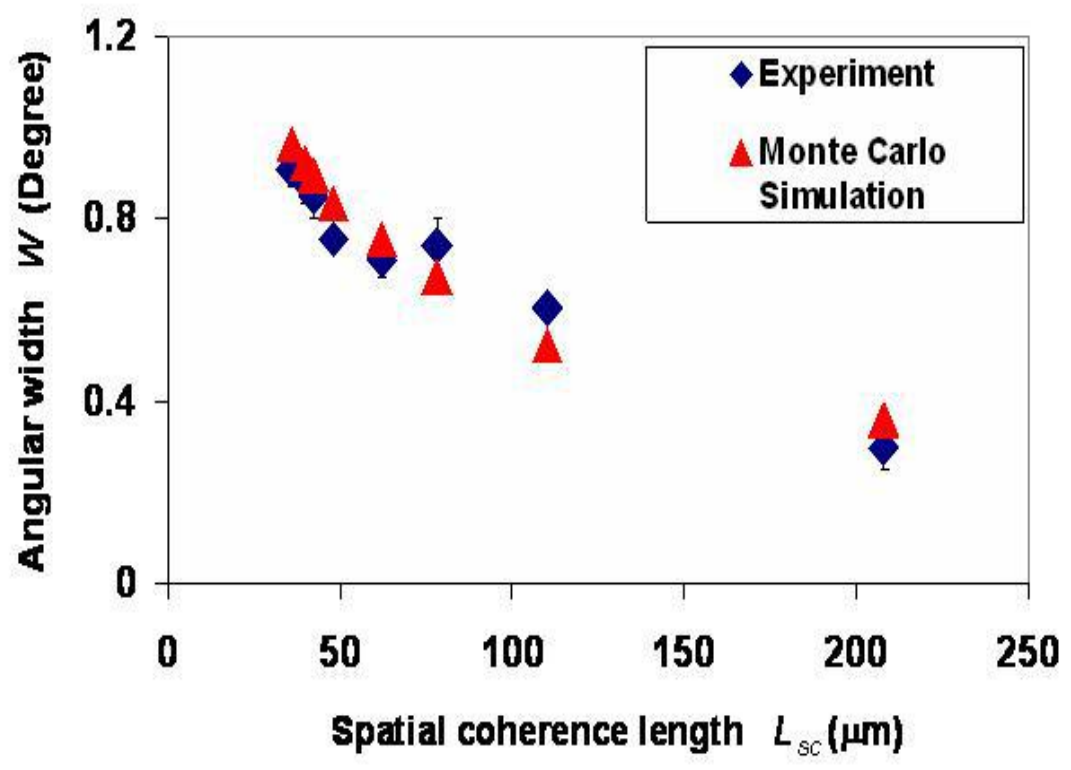

Figure 7: Comparison of angular width $W$ of the LEBS peaks obtained from Monte Carlo simulation with that of LEBS peaks from experiment $($ bead diameter $=0.89 \mu \mathrm{m})$ under low spatial coherence illumination $($ Xenon lamp, $\lambda=520 \mathrm{~nm})$.

We vary the transport mean free path $l_{s}^{*}$ from $500 \mu \mathrm{m}$ and $2000 \mu \mathrm{m}$ and the spatial coherence lengths from 30 $\mu \mathrm{m}$ and $220 \mu \mathrm{m}$. As a representative, we show here the results obtained from the sample of $l_{s}^{*}=2000 \mu \mathrm{m}$ and bead diameter of $0.89 \mu \mathrm{m}$. As shown in Fig. 6, LEBS peak predicted by Monte Carlo simulations is in excellent agreement with the peak recorded in the experiment $\left(L_{s c}=42 \mu \mathrm{m}\right)$. Further, the LEBS peak obtained from higher exit angles (asterisk) does not accurately model the LEBS peak as it is insensitive to the low orders of scattering. On the other hand, 
the broadening of LEBS peak can be accurately obtained only at low exit angles (circle) as it is more sensitive to the low orders of scattering. The simulations are further verified for eight other values of $L_{s c}$ varying from 35 to $220 \mu \mathrm{m}$ (Fig. 7). The error bars in the curves are the standard error obtained from 3 different sets of experiments conducted at different spatial coherence lengths. As shown in Fig. 7, the widths of LEBS peaks predicted by the Monte Carlo simulations are in excellent agreement with those determined in the experiment. This confirms that the Monte Carlo simulation can be used to model LEBS.

\section{CONCLUSIONS}

In conclusion, we have demonstrated for the first time that the photon random walk model can be used to model low coherence enhanced backscattering from a weakly scattering medium, such as biological tissue. We showed that LEBS predicted by the simulations was in excellent agreement with the experimental data. Furthermore, we have demonstrated that the exit angles of the photons, which are typically neglected in modeling of conventional EBS, play key role in modeling of LEBS. In particular, LEBS peak intensity is proportional to the Fourier transform of the radial distribution of light intensity on the surface of a sample $(P(r))$. For low order scattering $P(r)$ is sensitive to the exit angles of the photons, which in turn depend on the depth from which the photons are collected. Thus, we conclude that in order to model LEBS, it is crucial to account for the effect of exit angle on $P(r)$.

\section{ACKNOWLEDGEMENT}

This study was supported in part by National Institutes of Health grant, R01 EB003682, R01 CA112315 and National Science Foundation grant BES-0238903.

\section{REFERENCES}

1. Y. Kuga and A. Ishimaru, "Retroreflectance from a dense distribution of spherical particles," Journal of the Optical Society of America A - Optics Image Science and Vision 1, 831-835 (1984).

2. M. B. van der Mark, M. P. van Albada, and A. Lagendijk, "Light scattering in strongly scattering media: multiple scattering and weak localization," Physical Review B 37, 3575-3592 (1988).

3. P.E. Wolf, G. Maret, E. Akkermans, and R. Maynard, "Optical coherent backscattering by random media: an experimental study," Journal de Physique 49, 63-75 (1988).

4. R. Lenke and G. Maret, "Magnetic field effects on coherent backscattering of light," European Physical Journal B 17, 171-185 (2000).

5. M. Tomita and H. Ikari, "Influence of finite coherence length of incoming light on enhanced backscattering," Physical Review B 43, 3716 (1991).

6. A. Dogariu, J. Uozumi, and T. Asakura, "Enhancement of the backscattered intensity from fractal aggregates," Waves Random Media 2, 259-263 (1992).

7. D. S. Wiersma, M. P. van Albada, and A. Lagendijk, "Coherent backscattering of light from amplifying random media," Physical Review Letters 75, 1739-1742 (1995).

8. Y. L. Kim, Y. Liu, R. K. Wali, H. K. Roy, M. J. Goldberg, A. K. Kromin, K. Chen, and V. Backman, "Simultaneous measurement of angular and spectral properties of light scattering for characterization of tissue microarchitecture and its alteration in early precancer," IEEE Journal of Selected Topics in Quantum Electronics. 9, 243-256 (2003).

9. G. Yoon, D. N. G. Roy, and R. C. Straight, "Coherent backscattering in biological media: measurement and estimation of optical properties," Applied Optics 32, 580-585 (1993).

10. K. M. Yoo, G. C. Tang, and R. R. Alfano, "Coherent backscattering of light from biological tissues," Applied Optics 29, 3237-3239 (1990).

11. K. M. Yoo, F. Liu, and R. R. Alfano, "Biological materials probed by the temporal and angular profiles of the backscattered ultrafast laser pulses," Journal of Optical Society of America B 7, 1685-1693 (1990).

12. Y. L. Kim, Y. Liu, V. M. Turzhitsky, H. K. Roy, R. K. Wali, and V. Backman, "Coherent backscattering spectroscopy," Optics Letters 29, 1906-1908 (2004).

13. Y. L. Kim, Y. Liu, R. K. Wali, H. K. Roy, V. Backman, "Low-Coherent Backscattering Spectroscopy for Tissue Characterization," Applied Optics, 44 (3), 366-377 (2005). 
14. Y. L. Kim, Y. Liu, V. M Turzhitsky, R. Wali, H. Roy, and V. Backman, "Depth-resolved Low-coherent Backscattering in tissue," Optics Letters 30, 741-743 (2005).

15. R. Lenke, R. Tweer and G. Maret, "Coherent backscattering of turbid samples containing large Mie spheres," Journal of Optics A - Pure and Applied Optics 4, 293-298 (2002).

16. M. H. Eddowes, T. N. Mills and D. T. Delpy, "Monte-Carlo simulations of coherent backscatter for identification of the optical coefficients of biological tissue in-vivo," Applied Optics 34, 2261-2267 (1995).

17. R. Lenke and G. Maret, Multiple scattering of light: coherent backscattering and transmission, Scattering in Polymeric and Colloidal Systems, W Brown and K Mortensen, eds. (London, Gordon and Breach, 2000), pp. 1-73.

18. M. Born and E. Wolf, Principles of Optics: Electromagnetic Theory of Propagation, Interference and Diffraction of Light, $7^{\text {th }}$ ed. (Cambridge University, Cambridge, UK, 1999), pp. 572-580.

19. E. Akkermans, P. E. Wolf and R. Maynard, "Coherent backscattering of light by disordered media: Analysis of peak line shape", Physical Review Letters 56, 1471-1474 (1986). 